\title{
Neurovascular complications of heterotopic ossification following spinal cord injury
}

\author{
$\mathrm{S}$ C Colachis III MD, ${ }^{1} \mathrm{D} \mathrm{M}$ Clinchot $\mathrm{MD},{ }^{2} \mathrm{D}$ Venesy $\mathrm{MD}^{3}$ \\ ${ }^{1}$ Assistant Professor, ${ }^{2}$ Chief Resident, ${ }^{3}$ Assistant Professor, Department of Physical \\ Medicine and Rehabilitation, The Ohio State University, Dodd Hall, 480 West Ninth \\ Avenue, Columbus, Ohio 43210, USA.
}

Compression of neurovascular structures from heterotopic ossification can result
in neurological and vascular sequelae. Three cases of neurovascular compression
due to heterotopic ossification illustrate the potential for neurovascular com-
pression resulting from this condition and underscore the importance of recog-
nising this uncommon, but notable complication following spinal cord injury.
Keywords: heterotopic ossification; neurovascular compression; spinal cord injury.

\section{Introduction}

Heterotopic ossification ( $\mathrm{HO})$ is a frequent complication following spinal cord injury (SCI). The occurrence in this population ranges from $16 \%$ to $53 \%$, depending upon the study design and the methods of detection employed. ${ }^{1-6}$ Although $\mathrm{HO}$ is often observed as an incidental finding on plain radiographs, ${ }^{4,6,7}$ progression of this condition can result in significant restriction in joint motion and ankylosis. ${ }^{4,6,7,8-10}$ Other reported sequelae include the development of pressure ulcerations, ${ }^{11}$ painful arthrosis, ${ }^{12}$ and malignant transformation. ${ }^{13}$

Compression of nervous or vascular structures by developing heterotopic bone has been reported following traumatic brain injury, ${ }^{11,14,17}$ femoral and acetabular fractures, ${ }^{18-20}$ muscle trauma, ${ }^{21}$ and extensive burns. ${ }^{11,17,22,23}$ The occurrence of neurovascular compression in the SCI population, however, is exceedingly rare. The following case reports illustrate the potential for neurovascular compromise by heterotopic bone formation and permits discussion regarding our experience with this uncommon complication.

\section{Case reports}

\section{Case 1}

A 21 year old male hurled himself into a door on may 30, 1991 resulting in immediate incomplete quadriplegia. He sustained a C6 burst fracture which required an anterior $\mathrm{C} 6$ corpectomy, a C5/C6 diskectomy, and an anterior interbody fusion using a right iliac crest bone graft. He was admitted for SCI rehabilitation approximately 2 weeks after injury. At the time of his admission, his diagnosis was C5 motor and sensory incomplete quadriplegia (Frankel D). By the end of the first week of rehabilitation, he was ambulating short distances. Ten days after admission, he noticed the onset of pain and burning paresthesiae in the anterior aspect of both thighs, increased with hip flexion. A 3-phase bone scan performed at that time showed a focal area of increased activity in the region of the right iliac crest donor site. The images of the hips were normal. Mild elevations in the serum alkaline phosphatase (116 units/liter) and SGOT levels were noted at that time.

He actively participated in the rehabilitation program despite continued thigh pain and paresthesiae. The condition improved with low dosages of amitriptyline and his hip range of motion remained normal. However, on July 12, 1991 he complained of significantly increased right thigh pain. This increased pain was associated with an acute decrease in right quadriceps strength from a grade of $4 / 5$ to $2 / 5$. There was additional loss of sensation in the femoral nerve distribution; no other motor or sensory abnormalities were observed. Electromyography of the right lower limb demonstrated membrane irritability and loss of motor units in the femoral nerve distribution. The evoked potential elicited by proximal stimulation of the right femoral nerve was significantly decreased in comparison to that of the left. A computerized 
axial tomography (CAT) scan of the pelvis was subsequently performed which demonstrated an irregular hypodense region of peripheral ossification within the enlarged right iliopsoas muscle that displaced the right femoral neurovascular bundle medially (Fig 1). There was a similar appearance within the left iliopsoas muscle to a much lesser degree. A follow up 3-phase bone scan obtained 12 days later demonstrated evidence of active $\mathrm{HO}$ involving the soft tissues about both femurs. The serum alkaline phosphatase level was now markedly elevated (375 units/liter).

He was placed on indomethacin and underwent surgical exploration of the inguinal region in view of the deteriorating neurological status. During surgery, the bony roof above the femoral nerve was uplifted. $\mathrm{He}$ rapidly regained strength in the right quadriceps muscle to that level prior to the femoral nerve entrapment. By the time of discharge from rehabilitation, he was ambulating with the use of a cane. A repeat electromyographic examination performed in November 1991 showed decreased membrane irritability and a marked improvement in the number of motor units recruited.
There was still evidence of mild motor unit loss at that time.

\section{Case 2}

A 19 year old man suffered a SCI in February, 1991 while mud sliding. Diagnostic work-up revealed a C6 compression fracture with $\mathrm{C6}$ complete quadriplegia. He underwent anterior fusion from C5 to C7. During his rehabilitation hospitalization, he developed $\mathrm{HO}$ of the right hip as seen on pelvic films. In August 1991, he developed severe perineal pain associated with temperature elevation and leukocytosis. A CAT scan of the abdomen and pelvis performed on August 25, 1991 demonstrated soft tissue swelling associated with heterotopic bone formation anterior to the acetabulum and femoral head bilaterally. Heterotopic bone formation was seen in the left iliacus muscle as well as in the soft tissues surrounding the right iliac crest. A mild degree of femoral vein compression was reported. In addition, bilateral pleural effusions were observed. A duplex B-mode ultrasound of the lower limbs was normal. His perineal pain, fevers, and leukocytosis resolved, but the pleural effusions remained unchanged in size

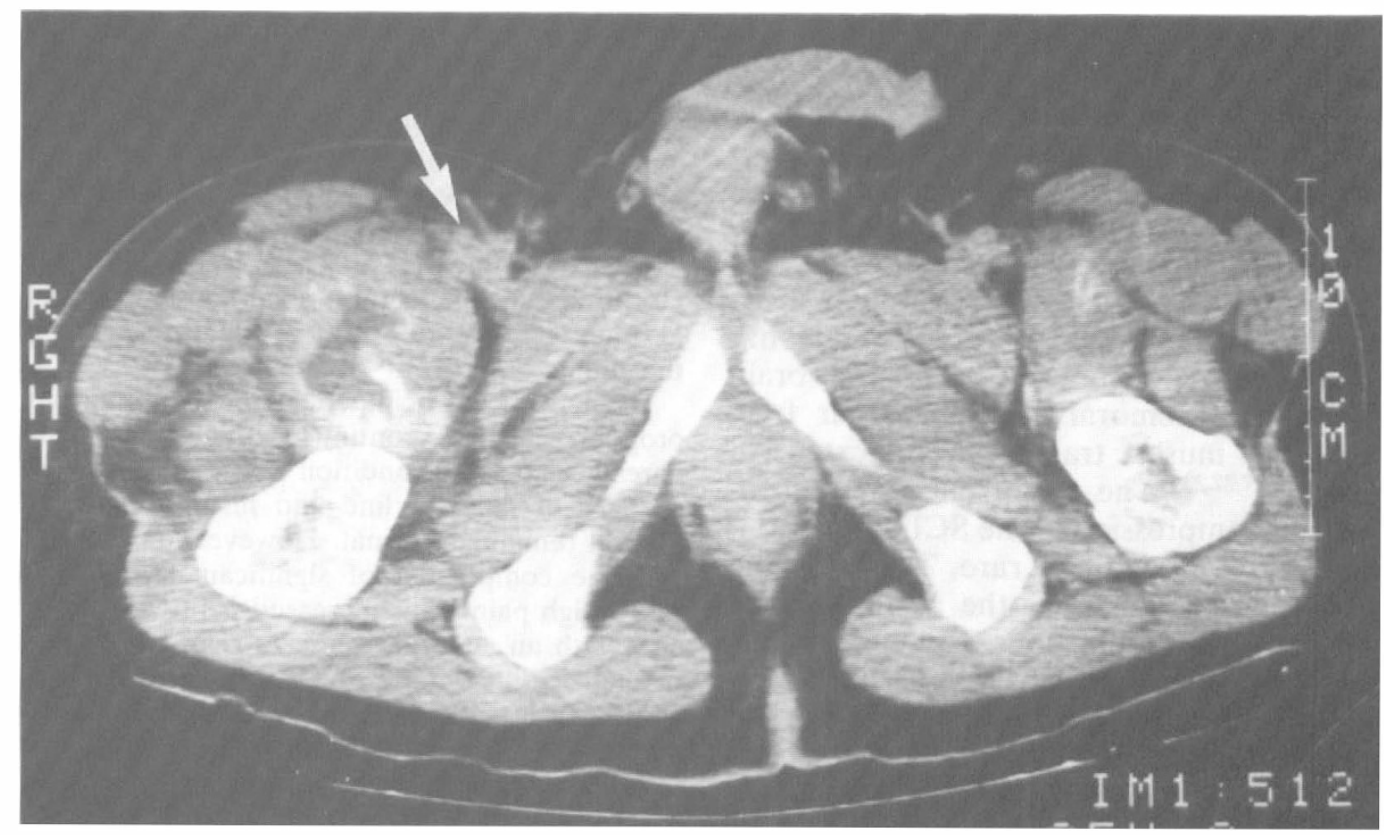

Figure 1 Computerized axial tomography (CAT) scanning of a section of the pelvis. An irregular hypodense region of peripheral ossification is seen within the enlarged right iliopsoas muscle which displaces the right femoral neurovascular bundle medially (arrow). There was a similar appearance within the left iliopsoas muscle to a much lesser degree. 
for the following 3 weeks. The pleural fluid collection was drained by thoracentesis on September 6, 1991. The fluid was consistent with an eosinophilic effusion.

Rehabilitation was continued, and he remained asymptomatic until 2 weeks later when he deveioped an episode of acute dyspnea and tachypnea. A ventilation-perfusion scan performed at that time indicated a high probability of a pulmonary embolus. Upon returning from the scan, he developed cardiopulmonary arrest, but was resuscitated immediately, and was transferred to the intensive care unit. A pulmonary angiogram revealed a large acute pulmonary embolus. An inferior vena cava filter was inserted and he was anticoagulated. He was readmitted in October of 1991 for completion of his rehabilitation without further complications.

\section{Case 3}

Acute left lower limb swelling developed in a 30 year old man with C6 complete quadriplegia in February, 1989. He was initially injured in a motor vehicle accident in 1980 . His history was significant for a hospitalization in 1987 for acute right lower limb swelling. Contrast venography performed at that time showed no evidence of deep venous thrombosis. However, fixed narrowing of the common femoral vein due to extrinsic compression was noted; a subsequent CAT scan of the region confirmed the large soft tissues mass. A 3-phase bone scan was consistent with active $\mathrm{HO}$ of the right hip. Since that time, he has been experiencing intermittent episodes of bilateral lower limb swelling.

In February 1989, he developed acute persistent swelling of the left lower limb. Contrast venography demonstrated a non thrombotic obstruction at the level of the femoral vein associated with thrombus involving the small collateral muscular branches (Fig 2). The study was repeated with the patient repositioned, at which time better filling of the femoral vein occurred. A follow up CT scan revealed diffuse swelling of the muscle groups of the left thigh with focal involvment of the left iliopsoas muscle. Heterotopic bone was seen within the distal aspect of the left iliopsoas muscle; a 3-phase bone scan confirmed activity of HO involving both hips. Swelling subsequently resolved, and he has had no significant recurrence since that time.

\section{Discussion}

Despite the high incidence of $\mathrm{HO}$ following injury to the spinal cord, ${ }^{1-5}$ compression of neurovascular structures by ectopic bone is quite uncommon. The large level of inflammation and vascularity involved in this acute process more often mimics other serious conditions such as thrombophlebitis, septic arthritis, cellulitis, hemorrhage, and bony tumors. ${ }^{6,7,10,24,25}$ In an extensive review of literature, case reports of $\mathrm{HO}$ leading to compression of the pelvic plexus or peripheral nerves in patients with SCI were not identified. Compression of vascular structures by $\mathrm{HO}$ was identified in only 3 patients with SCI. ${ }^{17,26}$ Orzel et al ${ }^{25}$ identified 2 cases of venous compression due to heterotopic bone confirmed by contrast venography, but they did not mention whether the patients had spinal injuries.

The potential for compression of neurovascular structures by $\mathrm{HO}$ is illustrated in the 3 cases presented. The patient in case 1 developed a proximal femoral nerve entrapment 6 weeks after he sustained a spinal cord injury. In case 2, ectopic bone was shown to compress the femoral vein as depicted during the CT scanning. Although duplex B-mode ultrasonography performed at the time was considered normal, the patient shortly thereafter developed an acute pulmonary embolus. The pulmonary embolus occurred more than 6 months after injury, long after the highest risk period for the development of this serious complication. ${ }^{27,28}$ Finally, a patient with long standing HO (case 3 ) developed venous compression as evidenced on venography; positional changes allowed flow of contrast material during one of the studies. He had evidence of thrombosis involving small intramuscular collateral vessels.

It is unclear why the prevalence of neurovascular compression is not greater than that observed, owing to the location of ectopic bone in this population. HO involving the hip most commonly occurs in line with the iliopsoas muscle, anterior and caudal to the hip joint; and along the medial aspect of the thigh in the region of the adductor musculature. . $^{3,4,7,8}$ The degree of local edema as well as the expanding bony mass can be significant, resulting in an increased risk for compression of neurovascular structures. The femoral and profunda vessels and the femoral nerve have been 

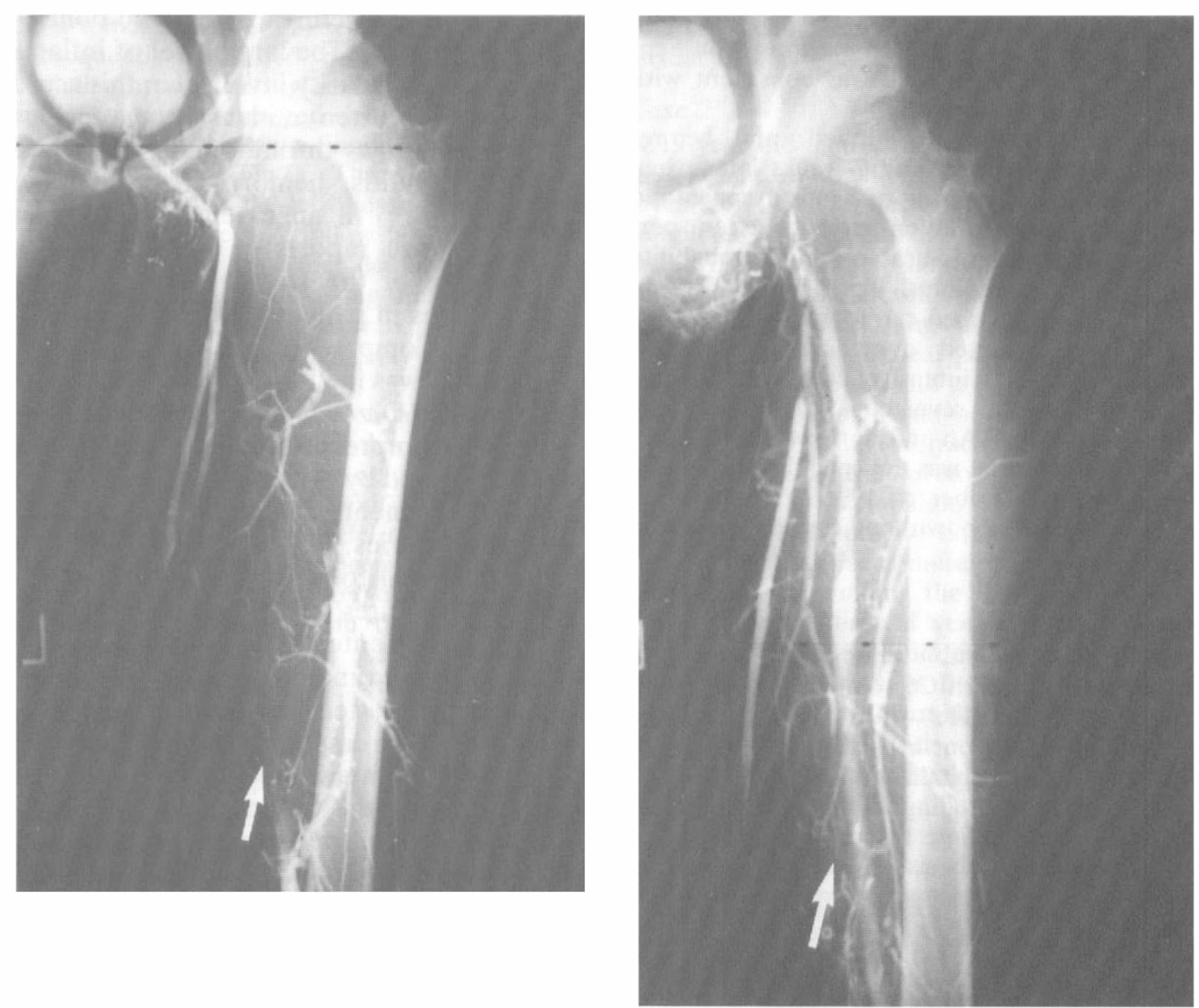

Figure 2 Contrast venography of the left lower limb. (a) a non thrombotic obstruction of the proximal superficial femoral vein with thrombus present in the intramuscular collateral branches; (b) a repeat study with the patient repositioned showing filling of the femoral vein.

observed during surgey to be enveloped in new bone. ${ }^{4}$

Several factors may account for the low prevalence of neurovascular compression observed. $\mathrm{HO}$ is often self limited, with minimal residual impairment. ${ }^{6,10}$ Partial or intermittent compression of neurovascular structures might not result in clinical findings of muscle atrophy, altered spasticity, or deep venous thrombosis. An example of this situation is presented in case 3 . Intermittent compression of venous return by ectopic bone more than likely produced the intermittent lower limb swelling. The venogram confirmed the presence of intermittent compression of venous flow concomitant with position, but showed no evidence of deep venous thrombosis involving the large vessels of the venous system. Progression of bone formation sufficient to compress nerves and nearby vascular structures resulting in clinically apparent features may result in ankylosis prior to neurovascular compromise.

Patients with complete spinal cord lesions will not experience pain, paresthesiae, or sensory loss commonly associated with compression neuropathies. ${ }^{29}$ In addition, these individuals develop loss of muscle mass ${ }^{30}$ making clinical features less apparent. Fortunately, the patient in case 1 had preservation of significant motor and sensory function which permitted recognition of peripheral nerve entrapment. With the growing 
numbers of individuals now surviving spinal cord injuries with incomplete lesions, ${ }^{31}$ we may see an increase in the incidence of entrapment neuropathies due to expanding ossification.

The most commonly reported entrapment neuropathy produced by $\mathrm{HO}$ involves the ulnar nerve at the elbow in patients with traumatic brain injury. ${ }^{14-17}$ Garland et al ${ }^{15}$ observed $\mathrm{HO}$ in 16 of 18 patients suffering tramatic brain injury. Two of these individuals developed ulnar nerve injury. In cases of ulnar nerve entrapment due to ossification, neurological recovery following decompression of the nerve had been variable, and is dependent upon the degree of neurapraxia or axonal loss present at the time of surgery. Ulnar nerve entrapment due to heterotopic bone formation has also occurred following extensive burns. ${ }^{11,14}$

The rapid development of ossification leading to peripheral nerve compression can be appreciated by the time course between the onset of symptoms and the activity of the heterotopic bone in case 1. At the time the patient first experienced symptoms, the serum alkaline phosphatase level, and 3-phase bone scan obtained were all normal. It was not until he developed objective neurological deterioration in the femoral nerve distribution that further diagnostic evaluation revealed extensive $\mathrm{HO}$ involving the inguinal region. Three-phase bone scans usually show active $\mathrm{HO} 2$ to 6 weeks prior to radiographic findings. ${ }^{8,11,25,32}$ In this case, however, significant bone formation was present less than 3 weeks following a normal 3-phase bone scan. This underscores the rapid development of extensive ossification concomitant with this condition. Several authors describe the sequence of clinical, laboratory, and radiological features observed during the development of $\mathrm{HO}, 3,7,8,24,25$ but detailed longitudinal studies clearly depicting the interval between onset of clinical signs, bone scan abnormalities, and ossification are unavailable. It is of interest that the calcification involving the iliopsoas muscle observed on the CT scan was not appreciated on the plain pelvic film taken the following day. By the time plain radiographs show clear evidence of $\mathrm{HO}$, ossification is extensive.
Most cases of DVT and pulmonary emboli (PE) occur during the first 2 to 3 months following SCI. ${ }^{27,28}$ The patient in case 2 had documented evidence of venous compression due to $\mathrm{HO}$ but a normal duplex scan. A CT scan performed at that time failed to identify any intraabdominal or pelvic thrombosis. He developed an acute PE shortly thereafter. The venous compression and subsequent PE occurred over 6 months after his spinal cord injury. Although he was at increased risk for the development of $\mathrm{PE}$, the occurrence shortly after the findings of venous compression and over 6 months after the onset of quadriplegia suggests a possible association between venous compression by the heterotopic bone and the development of an acute PE. It is recognized that a definite association is difficult, given the risk factors present after onset of paralysis. Duplex B-mode ultrasonography demonstrated a compressible vein despite the extensive $\mathrm{HO}$ in this patient. The technique can identify deep venous thrombosis as well as adjacent heterotopic bone, ${ }^{33}$ although the effectiveness of this technique for diagnosing both conditions has not been extensively studied.

The significant, but highly variable incidence of deep venous thrombosis (DVT) in the SCI population ${ }^{27,28,34}$ makes it difficult to draw any conclusions regarding the effects of $\mathrm{HO}$ upon this serious complication. Both conditions occur during the first few months following SCI. Acute $\mathrm{HO}$ often mimics DVT ${ }^{25,33}$ and once the diagnosis of DVT is determined, further evaluation of possible coexisting ectopic bone is less likely. In case 3, intermittent compression of the venous system of the lower limb was associated with thrombosis of intramuscular vessels. Although thrombosis of the large vessels was absent, it is intriguing to speculate that the involvement of smaller collateral vessels resulted from repeated intermittent compression of the large venous system. Recently, Haselkorn et $a l^{35}$ reported coexistent DVT and HO in a patient with transverse myelitis. They suggested that soft tissue swelling and impaired venous return may have lead to the development of DVT. Following this account, Varghese et $a l^{17}$ identified 2 patients with SCI in which 
femoral vein compression resulted from heterotopic ossification. Extensive venous thrombosis was present in one of the cases. We are unaware of any cases of intermittent compression of the venous system by heterotopic bone similar to that described in case 2 .
Compression of neurovascular structures from $\mathrm{HO}$ can result in definite neurological and vascular sequelae. It is important for physicians involved in the care of the SCI patient to recognize this uncommon but potentially serious complication of heterotopic bone formation.

\section{References}

1 Blane CE, Perkash I (1981) True heterotopic bone in paralyzed patient. Skeletal Radiol 7: 21-25.

2 Dejerine Mme, Ceiller A (1918) Para-osteo-arthropathies des paraplegiques par lesions medullaires (Étude clinique et radiographique). Ann Med 5: 497.

3 Freehafer AA, Yurick R, Mast WA (1966) Para-articular ossification in spinal cord injury. Med Serv J Can 22: 471-478.

4 Hardy AG, Dickson JW (1963) Pathological ossification in traumatic paraplegia. J Bone Joint Surg 45B: 76-87.

5 Liberson M (1953) Soft tissue calcifications in cord lesions. JAMA 152: 1010-1013.

6 Wharton GW, Morgan TH (1970) Ankylosis in the paralyzed patient. J Bone Joint Surg 52A: 105-112.

7 Kewalramani LS (1977) Ectopic ossification. Am J Phys Med 56: 99-121.

8 Garland DE (1991) A clinical perspective on common forms of acquired heterotopic ossification. Clin Orthop 263: 13-29.

9 Garland DE, Orwin JF (1989) Resection of heterotopic ossification in patients with spinal cord injuries. Clin Orthop 242: 169-176.

10 Hsu JD, Sakimura I, Stauffer ES (1975) Heterotopic ossification around the hip joint in spinal cord injured patients. Clin Orthop 112: 165-169.

11 Brooke MM, Heard DL, de Lateur BJ, Moeller DA, Alquist AD (1991) Heterotopic ossification and peripheral nerve entrapment: early diagnosis and excision. Arch Phys Med Rehabil 72: 425-429.

12 Good AE, Solsky MA, Gulati SM (1983) Heterotopic ossification simulating acute arthritis: a patient with stable, chronic, neurologic disease. J Rheum 10: 124-127.

13 Shanoff LB, Spira M, Hardy SB (1967) Myositis ossificans - evolution to osteogenic sarcoma. Report of a histologically verified case. Am J Surg 113: 537-541.

14 Cope R (1990) Heterotopic ossification. Southern Med J 83: 1058-1064.

15 Garland DE, O'Hollaren RM (1982) Fractures and dislocations about the elbow in the head-injured adult. Clin Orthop 168: $38-41$

16 Keenan MAE, Kauffman DL, Garland DE, Smith C (1988) Late ulnar neuropathy in the brain-injured adult. J Hand Surg 13A: 120-124.

17 Varghese G, Williams K, Desmet A, Redford JB (1991) Nonarticular complication of heterotopic ossification: a clinical review. Arch Phys Med Rehabil 72: 1009-1013.

18 Derian PS, Bibighaus AJ (1974) Sciatic nerve entrapment by ectopic bone after posterior fracture-dislocation of the hip. Southern Med J 67: 209-210.

19 Kleiman SG, Stevens J, Kolb L, Pankovich A (1971) Late sciatic-nerve palsy following posterior fracture-dislocation of the hip. J Bone Joint Surg 53A: 781-782.

20 Reinstein L, Eckholdt JW (1983) Sciatic nerve compression by preexisting heterotopic ossification during general anesthesia in the dorsal lithotomy position. Arch Phys Med Rehabil 64: 65-68.

21 Jones BV, Ward MW (1980) Myositis ossificans in the biceps femoris muscles causing sciatic nerve palsy. $J$ Bone Joint Surg 62B: 506-507.

22 Halls MJ (1988) Ulnar nerve entrapment. J Hand Surg 13A: 461.

23 Vorenkamp SE, Nelson TL (1987) Ulnar nerve entrapment due to heterotopic bone formation after a severe burn. J Hand Surg 12A: 378-380.

24 Jensen LL, Halar E, Little JW, Brooke MM (1988) Neurogenic heterotopic ossification. Am J Phys Med 66: $351-363$.

25 Orzel JA, Rudd TG (1985) Heterotopic bone formation: clinical, laboratory, and imaging correlation. $J$ Nucl Med 26: 125-132.

26 Bradleigh LH, Perkash A, Linder SH, Sullivan GH, Bhatt HK, Perkash I (1992) Deep venous thrombosis associated with heterotopic ossification. Arch Phys Med Rehabil 73: 293-294.

27 Walsh JJ, Tribe LR (1965) Phlebothrombosis and pulmonary embolism in paraplegia. Paraplegia 3: 209-213.

28 Watson N (1968) Venous thrombosis and pulmonary embolism in spinal cord injury. Paraplegia 6: 113-121.

29 Hallett M (1985) Electrophysiologic approaches to the diagnosis of entrapment neuropathies. Neurol Clinics 3: 531-541.

30 Ragnarsson KT (1988) Physiologic effects of functional electrical stimulation-induced exercises in spinal cord-injured individuals. Clin Orthop 233: 53-63. 
31 University of Alabama at Birmingham (1986) Spinal Cord Injury: The Facts and Figures.

32 Freed JH, Hahn H, Menter R, Dillon T (1982) The use of the three-phase bone scan in the early diagnosis of heterotopic ossification (HO) and in the evaluation of didronel therapy. Paraplegia 20: 208-216.

33 Scola FH, Parziale JR (1991) Ultrasonographic diagnosis of heterotopic ossification mimicking deep vein thrombosis. J Clin Ultrasound 19: 55-57.

34 Todd JW, Frisbie JH, Rossier AB, Adams DF, Als AV, Armenia RJ, et al (1976) Deep venous thrombosis in acute spinal cord injury: a comparison of ${ }^{125}$ I fibrinogen leg scanning, impedance plethysmography, and venography. Paraplegia 14: 50-57.

35 Haselkorn JK, Britell CW, Cardenas DD (1991) Diagnostic imaging of heterotopic ossification with coexistent deep-venous thrombosis in flaccid paraplegia. Arch Phys Med Rehabil 72: 227-229. 